\title{
Long-term Survival Models With Latent Activation Under a Flexible Family of Distributions
}

Vicente G. Cancho, Mário de Castro and Dipak K. Dey 
Table S1 Maximum likelihood estimates average (AMLE), standard deviation (SD) and square root of the mean squared error (RMSE) of the cured fractions $p_{0}^{(0)}$ (left, true value $=0.378$ ) and $p_{0}^{(1)}$ (right, true value $\left.=0.550\right)$ for data simulated from the geometric cure rate model under the first activation scheme.

\begin{tabular}{|c|c|c|c|c|c|c|c|c|}
\hline \multirow{2}{*}{$\begin{array}{l}\overline{\text { Fitted model }} \\
\text { Logarithmic }\end{array}$} & \multirow{2}{*}{$\begin{array}{l}\text { Activation } \\
\text { First }\end{array}$} & \multirow{2}{*}{$\begin{array}{c}n \\
200\end{array}$} & \multicolumn{2}{|c|}{ AMLE } & \multicolumn{2}{|c|}{$\mathrm{SD}$} & \multicolumn{2}{|c|}{ RMSE } \\
\hline & & & 0.388 & 0.534 & 0.0465 & 0.0615 & 0.0476 & 0.0634 \\
\hline & & 400 & 0.392 & 0.539 & 0.0342 & 0.0424 & 0.0371 & 0.0438 \\
\hline & & 800 & 0.392 & 0.538 & 0.0236 & 0.0288 & 0.0278 & 0.0313 \\
\hline & Last & 200 & 0.433 & 0.515 & 0.0431 & 0.0739 & 0.0701 & 0.0816 \\
\hline & & 400 & 0.438 & 0.519 & 0.0312 & 520 & 0.0680 & 0.0604 \\
\hline & & 800 & 0.436 & 0.521 & 0.0219 & 348 & 0.0624 & 0.0454 \\
\hline \multirow[t]{6}{*}{ Geometric } & First & 200 & 0.373 & 0.545 & 0.0513 & 0.0617 & 0.0515 & 0.0618 \\
\hline & & 400 & 0.377 & 0.549 & 0.0385 & 0.0424 & 0.0385 & 0.0424 \\
\hline & & 800 & 0.377 & 0.548 & 0.0263 & 289 & 0.0263 & 0.0290 \\
\hline & Last & 200 & 0.408 & 0.533 & 0.0484 & 0.0706 & 0.0571 & 0.0725 \\
\hline & & 400 & 0.412 & 0.537 & 0.0360 & 190 & 0.0500 & 0.0507 \\
\hline & & 800 & 0.411 & 0.537 & 0.0250 & 0.0331 & 0.0415 & 0.0354 \\
\hline \multirow[t]{6}{*}{$\overline{\text { Poisson }}$} & First & 200 & 0.367 & 0.553 & 0.0543 & 0.0624 & 0.0553 & 0.0624 \\
\hline & & 400 & 0.370 & 0.557 & 0.0412 & 29 & 0.0418 & 0.0434 \\
\hline & & 800 & 0.370 & 0.556 & 0.0280 & 0.0293 & 0.0290 & 0.0299 \\
\hline & Last & 200 & 0.385 & 0.548 & 0.0529 & 0.0673 & 0.0534 & 0.0673 \\
\hline & & 400 & 0.389 & 0.551 & 0.0399 & 0.0465 & 0.0415 & 0.0465 \\
\hline & & 800 & 0.388 & 0.551 & 0.0274 & 0.0315 & 0.0293 & 0.0316 \\
\hline \multirow[t]{3}{*}{ Mixture cure } & & 200 & 0.370 & 0.555 & 0.0555 & 0.0640 & 0.0560 & 0.0642 \\
\hline & & 400 & 0.373 & 0.558 & 0.0420 & 0.0442 & 0.0422 & 0.0450 \\
\hline & & 800 & 0.373 & 0.558 & 0.0287 & 0.0301 & 0.0290 & 0.0312 \\
\hline
\end{tabular}


Table S2 Maximum likelihood estimates average (AMLE), standard deviation (SD) and square root of the mean squared error (RMSE) of the cured fractions $p_{0}^{(0)}$ (left, true value $=0.378)$ and $p_{0}^{(1)}$ (right, true value $\left.=0.550\right)$ for data simulated from the geometric cure rate model under the last activation scheme.

\begin{tabular}{lllllllll}
\hline Fitted model & Activation & $n$ & \multicolumn{2}{c}{ AMLE } & \multicolumn{2}{c}{ SD } & \multicolumn{2}{c}{ RMSE } \\
\hline Logarithmic & First & 200 & 0.432 & 0.486 & 0.0545 & 0.0660 & 0.0770 & 0.0919 \\
& & 400 & 0.438 & 0.495 & 0.0380 & 0.0466 & 0.0715 & 0.0719 \\
& & 800 & 0.437 & 0.495 & 0.0263 & 0.0339 & 0.0646 & 0.0646 \\
& \multirow{2}{*}{ Last } & 200 & 0.391 & 0.541 & 0.0579 & 0.0698 & 0.0594 & 0.0703 \\
& & 400 & 0.393 & 0.545 & 0.0418 & 0.0479 & 0.0447 & 0.0481 \\
& & 800 & 0.393 & 0.548 & 0.0296 & 0.0340 & 0.0335 & 0.0340 \\
\hline Geometric & First & 200 & 0.411 & 0.495 & 0.0637 & 0.0694 & 0.0717 & 0.0884 \\
& & 400 & 0.417 & 0.504 & 0.0433 & 0.0475 & 0.0584 & 0.0660 \\
& \multirow{2}{*}{ Last } & 800 & 0.437 & 0.495 & 0.0263 & 0.0339 & 0.0646 & 0.0646 \\
& & 200 & 0.376 & 0.545 & 0.0651 & 0.0669 & 0.0651 & 0.0670 \\
& & 400 & 0.377 & 0.548 & 0.0476 & 0.0470 & 0.0476 & 0.0470 \\
& & 800 & 0.377 & 0.551 & 0.0331 & 0.0329 & 0.0331 & 0.0329 \\
\hline Poisson & \multirow{2}{*}{ First } & 200 & 0.396 & 0.513 & 0.0654 & 0.0662 & 0.0679 & 0.0756 \\
& & 400 & 0.400 & 0.520 & 0.0465 & 0.0471 & 0.0514 & 0.0560 \\
& \multirow{2}{*}{ Last } & 800 & 0.399 & 0.521 & 0.0320 & 0.0336 & 0.0383 & 0.0442 \\
& & 200 & 0.371 & 0.542 & 0.0704 & 0.0657 & 0.0706 & 0.0662 \\
& & 400 & 0.372 & 0.545 & 0.0514 & 0.0468 & 0.0516 & 0.0470 \\
& & 800 & 0.377 & 0.551 & 0.0337 & 0.0332 & 0.0337 & 0.0332 \\
\hline Mixture cure & & 200 & 0.382 & 0.531 & 0.0686 & 0.0655 & 0.0687 & 0.0682 \\
& & 400 & 0.383 & 0.535 & 0.0498 & 0.0468 & 0.0501 & 0.0491 \\
& & 800 & 0.383 & 0.537 & 0.0343 & 0.0331 & 0.0347 & 0.0353 \\
\hline
\end{tabular}


Table S3 Maximum likelihood estimates average (AMLE), standard deviation (SD) and square root of the mean squared error (RMSE) of the cured fractions $p_{0}^{(0)}$ (left, true value $=0.378$ ) and $p_{0}^{(1)}$ (right, true value $\left.=0.550\right)$ for data simulated from the Poisson cure rate model under the first activation scheme.

\begin{tabular}{lllllllll}
\hline Fitted model & Activation & $n$ & \multicolumn{2}{c}{ AMLE } & \multicolumn{2}{c}{ SD } & \multicolumn{2}{c}{ RMSE } \\
\hline Logarithmic & First & 200 & 0.406 & 0.534 & 0.0496 & 0.0580 & 0.0573 & 0.0601 \\
& & 400 & 0.408 & 0.531 & 0.0351 & 0.0397 & 0.0466 & 0.0439 \\
& & 800 & 0.408 & 0.531 & 0.0240 & 0.0285 & 0.0385 & 0.0340 \\
& Last & 200 & 0.430 & 0.534 & 0.0478 & 0.0677 & 0.0708 & 0.0695 \\
& & 400 & 0.429 & 0.531 & 0.0335 & 0.0474 & 0.0617 & 0.0511 \\
& & 800 & 0.427 & 0.532 & 0.0225 & 0.0348 & 0.0545 & 0.0392 \\
\hline Geometric & First & 200 & 0.388 & 0.544 & 0.0563 & 0.0583 & 0.0572 & 0.0586 \\
& & 400 & 0.406 & 0.543 & 0.0383 & 0.0455 & 0.0475 & 0.0460 \\
& \multirow{2}{*}{ Last } & 800 & 0.390 & 0.541 & 0.0269 & 0.0289 & 0.0295 & 0.0302 \\
& & 200 & 0.406 & 0.547 & 0.0543 & 0.0645 & 0.0610 & 0.0645 \\
& & 400 & 0.406 & 0.543 & 0.0383 & 0.0455 & 0.0475 & 0.0460 \\
& & 800 & 0.404 & 0.545 & 0.0256 & 0.0332 & 0.0366 & 0.0336 \\
\hline Poisson & \multirow{2}{*}{ First } & 200 & 0.377 & 0.553 & 0.0606 & 0.0587 & 0.0605 & 0.0588 \\
& & 400 & 0.379 & 0.549 & 0.0425 & 0.0408 & 0.0425 & 0.0407 \\
& \multirow{2}{*}{ Last } & 800 & 0.379 & 0.551 & 0.0287 & 0.0295 & 0.0288 & 0.0295 \\
& & 200 & 0.385 & 0.556 & 0.0599 & 0.0619 & 0.0604 & 0.0622 \\
& 400 & 0.386 & 0.552 & 0.0423 & 0.0436 & 0.0431 & 0.0436 \\
& & 800 & 0.385 & 0.554 & 0.0282 & 0.0318 & 0.0292 & 0.0320 \\
\hline Mixture cure & & 200 & 0.374 & 0.558 & 0.0627 & 0.0598 & 0.0628 & 0.0604 \\
& & 400 & 0.376 & 0.554 & 0.0441 & 0.0418 & 0.0441 & 0.0420 \\
& & 800 & 0.376 & 0.556 & 0.0295 & 0.0304 & 0.0296 & 0.0311 \\
\hline
\end{tabular}


Table S4 Maximum likelihood estimates average (AMLE), standard deviation (SD) and square root of the mean squared error (RMSE) of the cured fractions $p_{0}^{(0)}$ (left, true value $=0.378$ ) and $p_{0}^{(1)}$ (right, true value $\left.=0.550\right)$ for data simulated from the Poisson cure rate model under the last activation scheme.

\begin{tabular}{|c|c|c|c|c|c|c|c|c|}
\hline \multirow{2}{*}{$\begin{array}{l}\overline{\text { Fitted model }} \\
\text { Logarithmic }\end{array}$} & \multirow{2}{*}{$\begin{array}{l}\text { Activation } \\
\text { First }\end{array}$} & \multirow{2}{*}{$\begin{array}{c}n \\
200\end{array}$} & \multicolumn{2}{|c|}{ AMLE } & \multicolumn{2}{|c|}{$\mathrm{SD}$} & \multicolumn{2}{|c|}{ RMSE } \\
\hline & & & 0.431 & 0.515 & 0.0525 & 0.0628 & 0.0748 & 0.0719 \\
\hline & & 400 & 0.429 & 0.505 & 0.0365 & 0.0460 & 0.0627 & 0.0639 \\
\hline & & 800 & 0.429 & 0.512 & 0.0258 & 0.0320 & 0.0579 & 0.0499 \\
\hline & Last & 200 & 0.408 & 0.546 & 0.0554 & 0.0664 & .0632 & 0.0665 \\
\hline & & 400 & 0.405 & 0.540 & 0.0390 & 0.0475 & 0.0477 & 0.0485 \\
\hline & & 800 & 0.406 & 0.544 & 0.0269 & 0.0340 & 0.0389 & 0.0346 \\
\hline \multirow[t]{6}{*}{ Geometric } & First & 200 & 0.409 & 0.523 & 0.0595 & 0.0641 & 0.0675 & 0.0693 \\
\hline & & 400 & 0.408 & 0.515 & 0.0415 & 466 & 0.0515 & 0.0583 \\
\hline & & 800 & 0.408 & 0.521 & 0.0294 & 0.0324 & 0.0423 & 0.0436 \\
\hline & Last & 200 & 0.388 & 0.553 & 0.0621 & 0.0644 & 0.0630 & 0.0644 \\
\hline & & 400 & 0.387 & 0.546 & 0.0442 & 0.0463 & 0.0450 & 0.0464 \\
\hline & & 800 & 0.386 & 0.550 & 0.0306 & 0.0331 & 0.0317 & 0.0330 \\
\hline \multirow[t]{6}{*}{$\overline{\text { Poisson }}$} & First & 200 & 0.393 & 0.537 & 0.0634 & 0.0629 & 0.0652 & 0.0643 \\
\hline & & 400 & 0.393 & 0.529 & 0.0445 & 159 & 0.0469 & 0.0506 \\
\hline & & 800 & 0.391 & 0.534 & 0.0316 & 0.0323 & 0.0345 & 0.0360 \\
\hline & Last & 200 & 0.377 & 0.554 & 0.0677 & 0.0631 & 0.0677 & 0.0632 \\
\hline & & 400 & 0.377 & 0.547 & 0.0475 & 0.0456 & 0.0475 & 0.0456 \\
\hline & & 800 & 0.376 & 0.552 & 0.0299 & 0.0314 & 0.0299 & 0.0314 \\
\hline \multirow[t]{3}{*}{ Mixture cure } & & 200 & 0.380 & 0.548 & 0.0669 & 0.0626 & 0.0670 & 0.0625 \\
\hline & & 400 & 0.381 & 0.541 & 0.0472 & 0.0455 & 0.0472 & 0.0463 \\
\hline & & 800 & 0.379 & 0.546 & 0.0332 & 0.0321 & 0.0333 & 0.0324 \\
\hline
\end{tabular}


Table S5 Maximum likelihood estimates average (AMLE), standard deviation (SD) and square root of the mean squared error (RMSE) of the cured fractions $p_{0}^{(0)}$ (left, true value $=0.378$ ) and $p_{0}^{(1)}$ (right, true value $\left.=0.550\right)$ for data simulated from the mixture cure model.

\begin{tabular}{llccccccc}
\hline Fitted model & Activation & $n$ & \multicolumn{2}{c}{ AMLE } & \multicolumn{2}{c}{ SD } & \multicolumn{2}{c}{ RMSE } \\
\hline Logarithmic & First & 200 & 0.415 & 0.515 & 0.0501 & 0.0610 & 0.0625 & 0.0703 \\
& & 400 & 0.415 & 0.518 & 0.0337 & 0.0459 & 0.0503 & 0.0557 \\
& & 800 & 0.419 & 0.522 & 0.0261 & 0.0298 & 0.0487 & 0.0404 \\
& \multirow{2}{*}{ Last } & 200 & 0.413 & 0.533 & 0.0502 & 0.0684 & 0.0612 & 0.0704 \\
& & 400 & 0.416 & 0.534 & 0.0358 & 0.0494 & 0.0523 & 0.0517 \\
& & 800 & 0.419 & 0.536 & 0.0255 & 0.0338 & 0.0489 & 0.0363 \\
\hline Geometric & First & 200 & 0.395 & 0.525 & 0.0566 & 0.0619 & 0.0593 & 0.0668 \\
& & 400 & 0.396 & 0.528 & 0.0379 & 0.0462 & 0.0423 & 0.0511 \\
& \multirow{3}{*}{ Last } & 800 & 0.399 & 0.531 & 0.0299 & 0.0303 & 0.0367 & 0.0356 \\
& & 200 & 0.392 & 0.543 & 0.0566 & 0.0661 & 0.0584 & 0.0664 \\
& & 400 & 0.395 & 0.545 & 0.0400 & 0.0481 & 0.0436 & 0.0484 \\
& & 800 & 0.397 & 0.546 & 0.0296 & 0.0328 & 0.0354 & 0.0330 \\
\hline Poisson & \multirow{2}{*}{ First } & 200 & 0.382 & 0.537 & 0.0608 & 0.0623 & 0.0609 & 0.0636 \\
& & 400 & 0.383 & 0.540 & 0.0409 & 0.0463 & 0.0413 & 0.0474 \\
& \multirow{2}{*}{ Last } & 800 & 0.384 & 0.542 & 0.0324 & 0.0306 & 0.0332 & 0.0317 \\
& & 200 & 0.377 & 0.548 & 0.0620 & 0.0643 & 0.0619 & 0.0643 \\
& & 400 & 0.380 & 0.550 & 0.0428 & 0.0472 & 0.0428 & 0.0472 \\
Mixture cure & & 800 & 0.381 & 0.551 & 0.0329 & 0.0319 & 0.0330 & 0.0319 \\
& & 200 & 0.374 & 0.546 & 0.0636 & 0.0629 & 0.0637 & 0.0630 \\
& & 400 & 0.376 & 0.549 & 0.0430 & 0.0466 & 0.0430 & 0.0466 \\
& & 800 & 0.377 & 0.550 & 0.0320 & 0.0310 & 0.0320 & 0.0310 \\
\hline
\end{tabular}

